\title{
Research on Matters in Maintenance and Management of Ship Anchor
}

\author{
Wu Guoqiang \\ Tianjin Maritime College, Tianjin, 300350
}

Keywords: ship anchor; maintenance and management; matters needing attention

\begin{abstract}
Ship is an important means of transportation, and with the development of society, ships are also constantly changing. The ship anchor belongs to a very important deck machinery, therefore, it is of great significance to do a good job in the maintenance and management of the ship anchor. This paper probes into the matters needing attention in the maintenance and management of ship anchors, and concretely analyzes the maintenance and management of mechanical and hydraulic windlasses in practical work for reference.
\end{abstract}

Ship anchor is generally installed on the main deck of the front and back ship, whose main function is for the warping hawser of ship when dropping and weighing anchor, and it is a necessary navigational equipment to ensure the safe navigation and berthing of the ship. At present, ship anchors are widely used in China, and all kinds of ships need to be equipped with corresponding anchors, but ship anchors can be divided into three types: manpower windlasses, mechanical windlasses and hydraulic windlasses because of the different driving forms. With the continuous development of science and technology, the frequency of manpower windlasses is becoming smaller and smaller. At present, most of the ship's driving mode is mainly mechanical and hydraulic. Therefore, when exploring the matters needing attention in the maintenance and management of ship machinery, the main methods are mechanical windlasses and hydraulic windlasses, and the specific contents as follows.

\section{Maintenance and Management of Mechanical Windlasses in Practice Work}

\subsection{Composition of mechanical windlasses}

Mechanical windlasses is a kind of ship anchor, which is mainly driven by machinery. The main components of mechanical windlasses include guide wheel, anchor chain roller, master controller, electric control box, brake, worm and gear, motor, etc. [1]. In the maintenance and management of mechanical windlasses, what the first need to do is to master the its composition, only in that way can ensure the quality of maintenance and management as well as the its normal use.

\subsection{Matters in maintenance and management of mechanical windlasses}

Anchor is an indispensable part of the ship, and also an important instrument to ensure the safe navigation and berthing of the ship. Therefore, it is of great significance to do a good job in the maintenance and management of mechanical windlasses and ensure the normal operation of the anchors. [2]. In the maintenance and management of mechanical windlasses, it is mainly divided into two parts: electrical and mechanical maintenance and management. Hereinafter, the two specific maintenance and management will be explored:

Firstly, it is maintenance and management in the electrical part of the mechanical windlasses. The maintenance and management of the electrical part can be divided into three aspects: major overhauls, routine maintenance, daily repair and maintenance, and the specific maintenance and management work also need to start from these three aspects [3]. The first is major overhaul. The period of major overhaul is generally three to five years, and its specific work includes disassembly clean of motor of mechanical windlasses; check the safety of the circuit(short circuit, open circuit, loosening, disconnection, etc.);measure the insulation resistance of the coils to ensure that the insulation treatment meets the relevant requirements, and if the insulator appears the phenomenon 
of heat and scratch, it should be timely maintained to ensure the safety of the circuit; Replace bearing shaft; check the braking systems, etc. In addition to general maintenance items, the major overhauls also include that check and clean the main contact of the contactor and replace the damaged one; do a good job of relay protection, and check and maintain the voltage of electromagnetic brake; check the condition of the master controller, do a good job of insulation protection. Major overhaul is the process of extensive, thorough, comprehensive cleaning and overhaul of the anchors. In the process of overhaul, it needs to be paid more attention and carried out in accordance with relevant regulations. [4]. Second is routine maintenance. The main tasks of routine maintenance include: the removal of dirt and dust in the chassis and the inspection of various circuit components; check the insulation resistance and parts of the control box according to the relevant technical requirements and prescribed data to ensure that they meet the relevant requirements; check whether the earthing screw is loose, whether the earthing device is intact, whether there is damage in each circuit, whether the contactor will have a different current sound when working, etc. ;check whether the ammeter of the main order controller can work properly and the contacts are connected according to the related contact chart sequence, etc. If problems are found in the process of maintenance, timely maintenance and repair should be carried out. At the same time, in order to ensure safety, maintenance personnel need to take adequate protection measures and cut off the power supply before routine maintenance and repair. Third is daily repair and maintenance. Daily repair and maintenance is the basic to ensure the normal work of mechanical windlasses. In order to ensure the quality of maintenance, a special daily maintenance team should be set up, and the members of this team should have certain professional knowledge and skills and are competent for maintenance and repair work [5]. At the same time, formulate the scope of daily maintenance, work content, specific working hours, etc., to ensure work efficiency. At the same time, it is necessary to check the normal condition of the anchorage chassis, and if the temperature is found to be overheated, it is necessary to do the cooling work well. In the process of use, the anchor has a certain rated current. In order to ensure that the current does not exceed the rated current in the working process of the motor, it is necessary to measure the working current of the motor for daily repair and maintenance. Once the current is too large or has an abnormal smell, it needs to stop and check immediately, and well conduct the troubleshooting. Unlike routine maintenance and major overhaul, daily repair and maintenance is a basic work, which is the key to timely find problems and prevent major problems. Therefore, relevant management units should pay more attention to daily repair and maintenance work and implement relevant systems to ensure the quality of repair and maintenance. The final is the maintenance and management of the electrical controller and the master controller. The electrical controller and the master controller are the core of the electrical part of the mechanical windlasses and its maintenance and management work is mainly the setting of the relay and the check of the current relay contact. Among them, the relay setting needs to check the action current of the current relay. Under normal circumstances, the action current should be controlled at about $130 \%$ of the high-speed rated current of the motor. When at rated load condition, the motor needs to control the setting time at about 2 seconds, and to ensure that the time of medium-speed steady state to high-speed steady state is about 2.5 seconds The contact point of each current relay should be checked to ensure the minimum contact resistance and the specification of the thermal relay should be determined according to the motor, the shutoff should be controlled in about two minutes, and the setting situation should be determined according to the relevant equipment description and the actual engineering condition. At the same time, the electrical controller and the master controller also need regular routine maintenance to ensure that problems can be found and solved in time. Among them, routine repair cycle and major overhaul cycle can be determined according to the actual situation, which is usually for one month and three months.

The second is the maintenance and management of the mechanical part of the anchor. In addition to the electrical part, the maintenance of the mechanical part of the anchor is also very important. The maintenance and management of the mechanical part of the anchor mainly includes five parts. First, when the anchor works, it is necessary to check whether the transmission gear, bearing, 
countershaft and so on are roller slippery or not, whether the braking degree is normal and appropriate or not. Second, it is necessary to check the degree of wear of the brake belt and the normal contact surface to ensure that the clutch is flexible [6] and normal, and carry out the routine inspection towards the oil quality and oil level in the fuel tank of gear case to ensure compliance with the normal operation requirements. Third, before the work, the anchor needs test run to understand the no-load operation and the rotate speed of each gear. Fourth, in the process of work, if you encounter the need for halfway brake, in order to avoid burning out the brake belt, as soon as possible brake tight once. Fifth, reasonably control the speed of weighing and dropping anchor and avoid the long-time use of high-speed or medium speed gear, so as to avoid overload use of the anchor, causing damage to it. At the same time, after the brake time reaches to a certain extent, because long-term wear, the working spring length and air gap will increase with it, it will result in the reduction of braking pressure and affect the braking effect. Therefore, in the process of mechanical maintenance, it is also necessary to regularly adjust the working spring and air gap of brake according to the relevant specifications, to ensure that the brake can play its due role, thereby ensuring the normal operation and safety of the anchor. In addition, in the course of daily work, it also needs to pay more attention to check whether there exist heating phenomena, abnormal vibrations and sounds, the integrity and firmness of bolts, roller slippage between parts and components, idle speed, variable speed lubricating oil level, etc., during the operation of mechanical equipment, and clean at regular intervals and ensure the service life of the anchor by various means.

\section{Maintenance and Management of Hydraulic Windlasses in Practice Work}

Hydraulic windlass is another kind of common anchor at present. It is mainly composed of electric control system, hydraulic pipe system, hydraulic parts, hydraulic pump station, motor, sprocket wheel, brake, anchor chain wheel, support, base, etc. The maintenance and management of hydraulic windlasses is the key to ensure the normal work of the anchor. In the maintenance and management of hydraulic windlasses, it is also divided into electrical part and hydraulic part. Hereinafter, it will carry out specific exploration about the maintenance and management of the two parts.

The first is the maintenance and management of the electric part of hydraulic windlasses. Y / $\Delta$ starting is the driving mode of most hydraulic electric control systems. Compared with the control-driven mode of dynamic drive, the maintenance and management of this drive mode is more convenient and simple [7]. Therefore, in the maintenance and management of the electrical part of hydraulic windlasses, it is mainly divided into two parts: major overhaul and routine maintenance. The repair period of the two parts is once a year and one quarter respectively, and the specific period needs to be further determined according to the actual situation. In the process of major overhaul and routine maintenance, it mainly aims at cleaning and maintenance of electrical parts, such as related circuits, parts, insulation resistance, etc., and the specific contents are similar to the electrical parts of mechanical windlasses.

The second is the maintenance and management of hydraulic part of hydraulic windlasses. The maintenance and management of hydraulic part of hydraulic windlasses is an important part of the actual working process of the anchor, and also an important link to ensure its normal work. The oil tank, oil pump and drive motor of hydraulic windlasses are all placed in the room. At the same time, in the driving process of motor, it doesn't carry the brake and its speed is single. Therefore, the maintenance and management of hydraulic part of hydraulic windlasses are simple, which is mainly divided into two parts: daily management and regular special inspection. The main work of daily management includes five aspects: first, pay attention to check the setting value of hydraulic part, including the setting value of temperature alarm equipment, the setting value of liquid level and so on. At the same time, we also need to carry out regular inspection on heating equipment of hydraulic fuel tank, especially in winter, when the temperature is low, it is necessary to ensure the heating equipment of the fuel tank so as to ensure the normal operation of the anchor [8]. Second, check suction pressure, the operation of each component and whether there is abnormal fever or not, and once finding abnormal situation, it needs to immediately check to ensure normal operation and 
work. Third, check and clean the hydraulic system. Mainly check the leakage of the system, and in the process of cleaning, it needs to follow these basic principles of no metal chips, no rust, no moisture, no oil sludge, etc. Fourth, in the process of oil exchange, the fuel tank and related systems need to be thoroughly cleaned, and the new oil need to have a good filtration rate to ensure that there is no dirt and residue. Fifth, do a good job of external cleaning, to avoid dust into the internal and impact the efficiency of normal work. Regular special inspection includes three aspects: the first is quarterly special inspection of fuel tank filter, whose main work is the cleaning and inspection of fuel tank filter and air filter to ensure that the tank filter can work properly. The second is annual special inspection. Its job content includes quarterly special inspection, and on this basis, according to the specific requirements of the annual special inspection, it needs to carry out the general inspection of the anchor, including pressure check, relief valve inspection, safety valve inspection, etc. The third is special inspection every two years which includes quarterly special inspection and annual special inspection content, and on this basis, it also needs to clean, maintain and renew the tank and test hydraulic oil quality.

\section{Conclusions}

To sum up, ships are essential tools for maritime traffic and play an important role in people's daily life. The ship anchor is an important tool for weighing and dropping anchor, and it is also the key to ensure the safe navigation of the ship. Therefore, it is of great significance to well maintain and manage the ship anchor.

\section{References}

[1] Tao Ruhao. Simple Analysis about Matters Needing Attention in Maintenance and Management of Ship Anchor [J] Transportation Enterprise Management, 2015, 30(9)39-41.

[2] Tang Yuhua. Application of Frequency Converting Control in the Control of Electric Windlass of Engineering Ship [J] Machine China, 2015(17)84-86

[3] Wang Yingqian. Simple Analysis about Daily Maintenance and Management of Ship Hydraulic Equipment [J] Shandong Industrial Technology, 2016(22)255-255.

[4] Xia Huafeng, Zhang Zhongguo and Liu Yanjun. Study on Quantum Particle Swarm Optimization PID Algorithm for Control System of Ship Anchor [J] Electrotechnics Electric, 2016, 28(1)25-28.

[5] Zhang Bo and Zhao Chunhui. Study on Strength Calculation of Base and Deck Support Structure of Oil Tanker Anchor [J] Journal of Zhejiang International Maritime College, 2016, 10 (3): 3-7.

[6] Zhou Chuan, Simulation Study and Application of Ship Hydraulic Windlass and Cargo Winch System [D] Dalian Maritime University, 2017.

[7] Xia Huafeng, Zhang Zhongguo and Liu Yanjun. Application of Particle Swarm Fuzzy Control Technology in the Control System of Ship Anchor [J] Micromotors, 2015, 48(11)89-93.

[8] Chen Pan. Analysis about Strength Calculation of Anchor Base and Supporting Hull Structure of Bulk Carrier[J]Naval Architecture and Ocean Engineering, 2016,32(5)55-60. 